\title{
A Drawing System for Pole Diagram and Rose Diagram of Rock Mass Structural Surface
}

\author{
Gaojian Hu $\mathbb{1 D}^{1,2,3}$ \\ ${ }^{1}$ School of Civil Engineering, Shaoxing University, Shaoxing, Zhejiang 312000, China \\ ${ }^{2}$ Key Laboratory of Rock Mechanics and Geohazards of Zhejiang Province, Shaoxing, Zhejiang 312000, China \\ ${ }^{3}$ Zhejiang Collaborative Innovation Center for Prevention and Control of Mountain Geologic Hazards, Shaoxing, \\ Zhejiang 312000, China \\ Correspondence should be addressed to Gaojian Hu; hugaojian8@163.com
}

Received 4 June 2021; Accepted 21 July 2021; Published 30 July 2021

Academic Editor: Fazlullah Khan

Copyright (c) 2021 Gaojian Hu. This is an open access article distributed under the Creative Commons Attribution License, which permits unrestricted use, distribution, and reproduction in any medium, provided the original work is properly cited.

\begin{abstract}
The distribution of structural planes is complex and irregular. How to quickly and effectively obtain the occurrence and distribution characteristics of structural planes and improve the efficiency of data processing is a key technical issue in structural plane analysis. The paper combined fuzzy equivalent clustering algorithm, rose diagram drawing method, and $\mathrm{C}++$ language, developed a drawing system for the pole diagram and rose diagram of rock mass structural planes, and verified the practicability of the system through an engineering case analysis. In the article, the fuzzy equivalent clustering of the occurrence of structural planes, the drawing of pole diagram, and the statistical analysis of structural planes were realized based on the fuzzy equivalent clustering algorithm. The rapid drawing of the striking rose diagram, trending rose diagram, and dip rose diagram module were realized based on the rose diagram drawing method. The system integrated the structure plane pole diagram and the occurrence rose diagram together and realized the rapid analysis and drawing of the structure plane.
\end{abstract}

\section{Introduction}

In the analysis of rock slope stability, the occurrence of structural planes has a great influence on slope stability. How to quickly and effectively obtain the occurrence and distribution characteristics of structural planes is a key issue in the acquisition and analysis of structural planes.

At present, the commonly used methods for collecting structural plane occurrence include the survey line method, precise survey line method, sampling window method, and borehole core joint collection method. For example, Zhao et al. [1] used the survey line method to evaluate the stability of the underground surrounding rock of the Lingbao Luoshan Gold Mine. Chaminé et al. [2] measured the actual data of rock outcrops using the precise line method. Xu et al. [3] improved the statistical method of sampling window to obtain the structural surface data of granite outcrops. Manzoor et al. [4] used the measurement while drilling method to count the data of different structural planes in open-pit mines and established the relationships between drilling parameters and rock mass structure.

These measurement methods have shortcomings such as large workload, large error, and poor effect when applied in the field. At the same time, the obtained structural data is complex and irregular, which is very cumbersome and difficult to process manually. Therefore, an intelligent software system is needed to automatically realize the processing of structural data.

The pole diagram and rose diagram are commonly used statistical methods of structural plane occurrence. The rose diagram is a simple, clear, and intuitive basic geological map, which is widely used in characterizing the development degree and dominant orientation of structural surfaces. The rose diagram is simple and helpful to analyze the regional structure. It can be more clear in reflecting the direction of the main joints. For example, Wang et al. [5] realized the 
rapid analysis and recognition of the rose diagram of the structural plane based on the MATLAB computing platform. Dong et al. [6] used rose diagrams and pole diagrams to perform statistical analysis on the structural plane information of fractured rock masses.

In geological analysis, the joint rose diagram is usually plotted on the geological map according to the positions of the survey point to clearly reflect the relationship between the joints, folds, or faults in different structural parts. Among them, the strike rose diagram is mostly used in situations where the joint occurrence is relatively steep. For example, Tao et al. [7] used equatorial horizon projection and strike rose diagram to determine the dominant structural plane. Wei et al. [8] used the strike rose diagram method to calculate the structural plane information of the rock mass in the Beishan area.

The trend rose diagram and dip rose diagram are mostly used in situations where the joint occurrence changes greatly. For example, Jifeng et al. [9] realized the automatic drawing of the trend rose diagram with the help of the MATLAB program, which can quickly obtain structural information. Li et al. [10] took the tunnel face as the background and used the dip rose diagram method to realize the difference visualization analysis of the tunnel radial rock mass structure. The disadvantage of the occurrence rose diagram is that it is grouped only according to the distribution of occurrence. When there are multiple sets of structural planes for the same occurrence, the rose diagram of the occurrence cannot be fully represented. It is difficult to realize the intelligent drawing of large-scale structure surface data because the drawing method of the occurrence rose diagram is cumbersome. It needs to be repeated many times when there are multiple sets of structural planes for a certain occurrence. Therefore, it is necessary to realize the automatic drawing of the rose diagram of the occurrence state through a software program.

The pole diagram represents the projection of the poles of the measured structural plane onto the stereographic projection diagram. It is a graphical method that uses points and lines on a plane to analyze lines and surfaces in three-dimensional space. The pole diagram can be rotated and transformed to restore the joints and stratum occurrence before the structural failure. The pole diagram can simultaneously show the distribution of the tendency and dip of the structural plane, which is more scientific and accurate than the rose diagram method. For example, Zhao et al. [11] used the pole diagram to study the distribution characteristics of dominant structural planes. Zhong et al. [12] used a combination of the rose diagram and pole diagram to perform statistical analysis on structural planes and determined the dominant structural planes. But the illustration process of the pole diagram is quite tedious. When a pole map is manually completed, such as an isodensity map, it needs to go through the processes of casting points, density statistics, percentage conversion, drawing straight lines, etc., which is time-consuming and errorprone. The grouping of pole diagrams mainly relies on empirical methods, and the results lack objectivity. Therefore, statistical analysis methods and computer processing programs have gradually become important methods for drawing pole diagrams.

Cluster analysis is a method of statistically studying classification problems. It allocates all sample data to several clusters so that the sample data of the same cluster are gathered around the cluster center. The cluster analysis methods include systematic clustering method, ordered sample clustering method, dynamic clustering method, and fuzzy clustering method. Among them, the fuzzy equivalent clustering method has better advantages in processing structure. For example, Li et al. [13] proposed a new method for identifying local high-density samples. Hou et al. [14] carried out a cluster analysis of the dominant occurrence of rock mass structural planes based on a netting algorithm. $\mathrm{Li}$ et al. [15] clustered the structural planes of rock mass based on the ant colony clustering method, which effectively realized the grouping of structural planes. Jun et al. [16] proposed an improved fast clustering analysis method. Guo et al. [17] introduced the firefly algorithm and the fuzzy $c$ means algorithm to accurately obtain the discontinuity information of the rock mass. Bei et al. [18] used the fuzzy clustering method to process the structural surface information of rock masses and obtained satisfactory results. Through the equivalent fuzzy clustering method, the cluster analysis of structural plane occurrence can be realized. The analysis result can accurately reflect the actual distribution of the data and obtain the precise occurrence distribution and data of the structural plane. Cluster analysis overcomes the subjectivity of human experience grouping. Therefore, writing a computer program system can quickly and accurately obtain structural plane occurrence information based on the fuzzy equivalent clustering analysis method.

Both the rose diagram and pole diagram can reflect the different aspects of the structural plane. There are more or fewer problems in the application and drawing of a single graphical method. If the amount of structural data obtained by field measurement is too large, there will be cumbersome and time-consuming processing problems. Therefore, if the two drawing methods can be integrated into one drawing system, the rose diagram and the pole diagram can be drawn at the same time, which will bring great benefits to the analysis of the occurrence of structural surfaces. Although scholars at home and abroad have realized the recognition of structural plane occurrences by writing programs, few scholars have formed it into a systematic and intelligent software system.

The article developed a drawing system for the pole map and occurrence rose diagram of rock mass structural plane based on fuzzy equivalent clustering algorithm and $\mathrm{C}++$ language programming to realize the rapid and intelligent drawing of rock mass structural plane.

\section{System Composition and Theoretical Basis}

The paper programming developed a drawing system of the pole map and rose diagram of rock mass structural plane based on fuzzy equivalent clustering analysis method, $\mathrm{C}++$ language, and the method of drawing rose diagram of structural plane occurrence. The paper combined theoretical 
algorithms and software system development to provide a drawing system for pole diagrams and rose diagrams of rock mass structural planes.

The system included 8 modules: the import module of data, the calculation module of the fuzzy equivalent clustering algorithm, the drawing module of pole diagram, the statistical analysis module of the structural plane, the output module of data, the drawing module of the striking rose diagram, the drawing module of trending rose diagram and drawing module of dip rose diagram. The system flow chart is shown in Figure 1.

The theoretical basis and realization functions of each module of the system were as follows.

2.1. Import Module of the Data. This module was used to import the structural plane data obtained by digital photogrammetry into the software system.

\subsection{Calculation Module of Fuzzy Equivalent Clustering} Algorithm. This module realized the fuzzy equivalent clustering of structural plane occurrence based on the fuzzy equivalent clustering algorithm. The process was as follows.

Supposing the number of the measured samples of the structural plane was $N$, and the $i$-th sample was represented as $\left(x_{i 1}, x_{i 2}\right) . x_{i 1}$ was the inclination of the structural plane, and $x_{i 2}$ was the dip of the structural plane. The fuzzy relation matrix $R$ was as follows:

$$
R=\left[\begin{array}{cccc}
r_{11} & r_{12} & \cdots & r_{1 N} \\
r_{21} & r_{22} & \cdots & r_{2 N} \\
\vdots & \vdots & \vdots & \\
r_{N 1} & r_{N 2} & \cdots & r_{N N}
\end{array}\right] .
$$

The element $r_{i j}$ in the matrix was the similarity coefficient between the $i$-th sample and the $j$-th sample, which represents the degree of similarity. The larger the $r_{i j}$, the more similar the sample $i$ and the sample $j$.

The calculation of the similarity coefficient $r_{i j}$ :

$$
r_{i j}= \begin{cases}1, & i=j, \\ 1-c\left(\left|x_{i 1}-x_{j 1}\right|+\left|x_{i 2}-x_{j 2}\right|\right), & i \neq j .\end{cases}
$$

In (2), $i=1,2, \ldots, N ; j=1,2, \ldots, N, c$ was the calculation parameter $(0 \leq c \leq 1)$, and the value of $c$ was appropriately selected to make $r_{i j}$ spread out in $[0,1]$.

Solve the closure $t(R)$ :

$$
\begin{gathered}
R^{2}=R R, \\
R^{4}=R^{2} R^{2}, \\
\cdots .
\end{gathered}
$$

Carry out structure plane grouping judgment. The steps of fuzzy matrix multiplication were similar to those of ordinary matrix multiplication. The difference was that instead of multiplying the two items first and then adding them, it took the smaller and then the larger. If $C=A B$, then the elements in $C$ conform to $\max _{k}\left(\min \left(a_{i k}, b_{i k}\right)\right)=\mathrm{V}_{k}$ $\left(a_{i k} \wedge b_{k j}\right)$. The $n$-level fuzzy relation matrix $R$ was the continuous multiplication of $n$ Rs. That is,

$$
\begin{aligned}
\max _{k}\left(\min \left(a_{i k}, b_{i k}\right)\right) & =\underset{k}{\vee}\left(a_{i k} \wedge b_{k j}\right), \\
R^{n} & =R^{n+1}=R^{n+2}=\cdots, \\
t(R) & =R^{n} .
\end{aligned}
$$

Take the cut-set level $\lambda \in[0,1]$, if $r_{i j} \geq \lambda$, then the structural planes $i$ and $j$ belong to the same category. That is,

$$
r_{i j} \geq \lambda
$$

2.3. Drawing Module of the Pole Diagram. According to the clustering results and the structural plane grouping results, the pole diagram of the structural plane was drawn. The process was as follows.

The lower hemisphere equal-angle projection method was used to draw the pole diagram.

The joint occurrence data represented by inclination $\alpha_{d}$ and $\operatorname{dip} \beta_{d}$ were converted into the joint occurrence data represented by the unit normal vector. Assuming that $\alpha_{n}$ and $\beta_{n}$ were the tilt direction and tilt angle of the unit normal vector of the structural plane. The unit normal vector for any structural surface was expressed as $X=\left(x_{1}, x_{2}, x_{3}\right)$, and each point on the hemispherical surface corresponded to a joint occurrence. The formula was as follows:

$$
\begin{aligned}
& X=\left(x_{1}, x_{2}, x_{3}\right), \\
& x_{1}=\cos \alpha_{n} \cos \beta_{n} \\
& x_{2}=\sin \alpha_{n} \cos \beta_{n} \\
& x_{3}=\sin \beta_{n} \\
& \alpha_{n}=\alpha_{d}+180^{\circ}, \quad \alpha_{d} \leq 180 \\
& \alpha_{n}=\alpha_{d}-180^{\circ}, \quad \alpha_{d} \geq 180 \\
& \beta_{n}=90^{\circ}-\beta_{d} \\
& \alpha_{d} \in(0,360), \\
& \beta_{d} \in(0,90) .
\end{aligned}
$$

The occurrence data of the structural plane expressed by the unit normal vector was obtained.

The coordinates $x_{n}$ and $y_{n}$ of $A^{\prime}$ on the stereographic projection diagram were calculated based on the normal occurrence data of the structural plane and the stereographic projection principle diagram. Among them, point $A^{\prime}$ was the equatorial projection of the plane normal. The formula was as follows:

$$
\begin{gathered}
r=\tan \frac{\beta_{d}}{2}, \\
\left\{\begin{array}{l}
x_{n}=r \sin \left(\alpha_{n}\right), \\
y_{n}=r \cos \left(\alpha_{n}\right) .
\end{array}\right.
\end{gathered}
$$




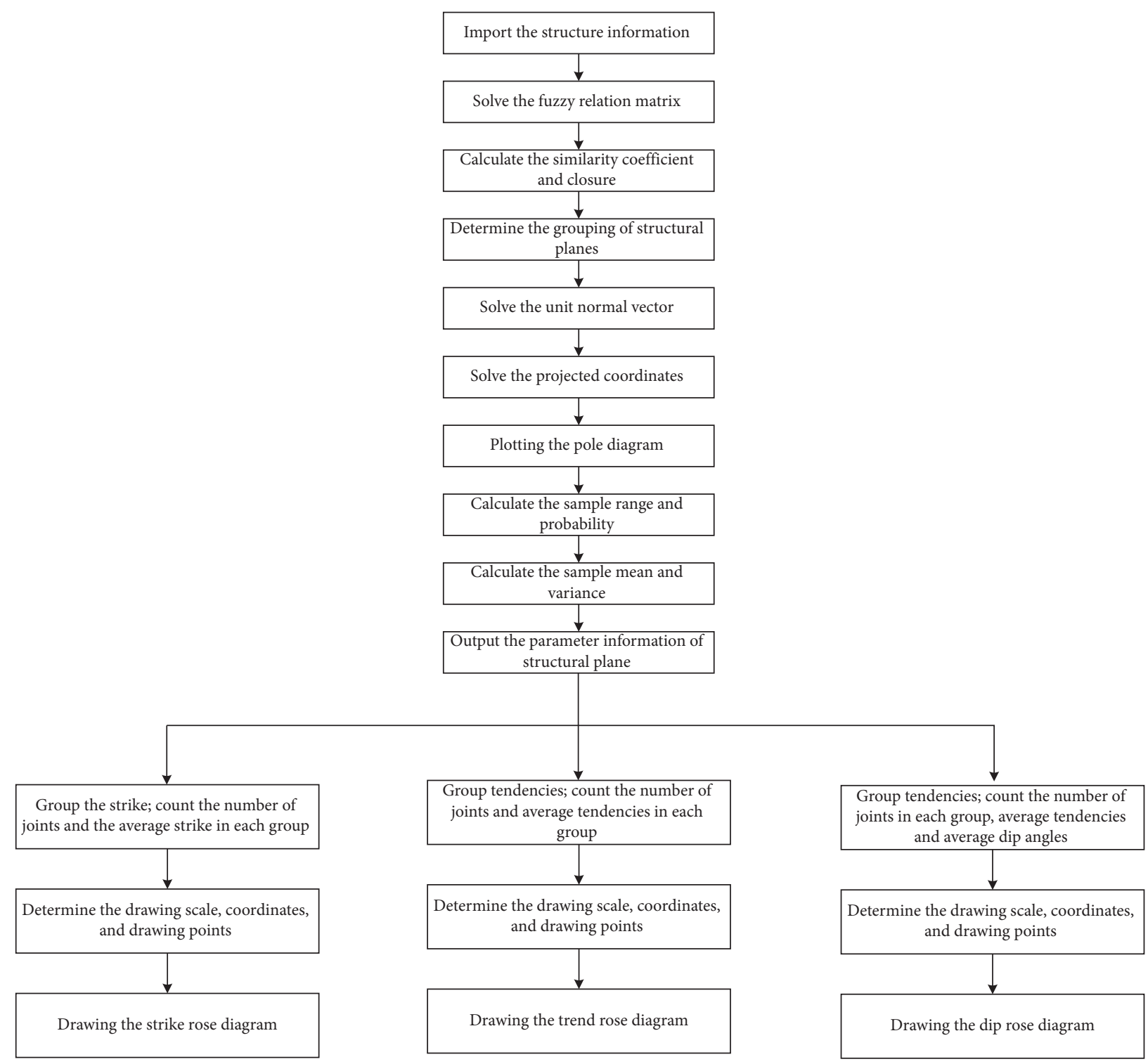

FIgURE 1: The flow chart of the system.

The stereographic projection coordinate points of all structural surface normals were solved.

A base circle whose diameter was unit length was drawn, and the vertical and horizontal diameters were drawn, and $E$, $S, W$, and $N$ directions were marked.

The stereographic projection coordinates of all structural surfaces were drawn on the base circle diagram. The pole diagram of the structural plane was drawn.

\subsection{Statistical Analysis Module of the Structural Plane.} This module was used for the statistical analysis of the structural planes after clustering to obtain the mean and variance of the inclination, dip, trace length, spacing, and rock bridges of each group of structural planes. The process was as follows.

The sample partition interval $m$ was determined firstly.

The sample range $\bar{R}$ was solved according to the following formula:

$$
\bar{R}=\max \left\{x_{i}\right\}-\min \left\{x_{i}\right\} .
$$

Each partition interval was calculated according to the following formula:

$$
M_{m}=\left\{\min \left\{x_{i}\right\}+\frac{\bar{R}}{7} \cdot m, \min \left\{x_{i}\right\}+\frac{\bar{R}}{7} \cdot(m+1)\right\} .
$$

The probability of the sample falling in each partition interval was determined. The method was to first use the computer loop language to count the number of samples $N_{m}$ that fall in each interval, and then combine the total number of samples $N$ to calculate the sample number probability $P_{m}$ :

$$
P_{m}=\frac{N_{m}}{N}
$$

The sample mean was solved according to the following formula: 


$$
\bar{x}=\frac{1}{N} \sum_{i=1}^{n} x_{i} .
$$

The sample variance $S^{2}$ was solved according to the following formula, where $S$ was the standard deviation:

$$
S^{2}=\frac{1}{N-1} \sum_{i=1}^{n}\left(x_{i}-\bar{x}\right) \text {. }
$$

According to the probability value $P_{m}$, the probability distribution patterns of the inclination, dip, trace, spacing, and rock bridges of each group of structural planes were drawn automatically.

2.5. Output Module of the Data. The classification information of structural planes was output, including the mean and variance of the inclination, dip, trace, spacing, and rock bridges of each group of structural planes.

2.6. Drawing Module of the Rose Diagram. The drawing module of the rose diagram includes the strike rose diagram, the trend rose diagram, and the dip rose diagram. The drawing module of the trend rose diagram was taken as an example to clarify its theoretical basis. The process was as follows:

The joints were sorted according to the size of the tendency data and grouped every $\theta=10^{\circ}$. Each group was named as $D_{j}$. The formula was as follows:

$$
\begin{aligned}
D_{j} & =\left\{\theta, \theta+9^{\circ}\right\}, \\
\theta & =10(j-1), \\
j & \in(1,36) .
\end{aligned}
$$

The number of joints in each group was named $S_{D}$, and the average tendency of each joint was named $\overline{D_{j}}$. Calculate $\overline{D_{j}}$ according to the following formula:

$$
\begin{aligned}
& \overline{D_{j}}=\frac{\text { the sum of the structure of the } D_{j} \text { group }}{S_{D_{j}}}, \\
& j \in(1,36) .
\end{aligned}
$$

According to the size of the drawing and the number of each group of joints, a certain length of line segment was selected to represent a group of joints, and the scale of the line segment $L_{d}$ was determined.

The group of joints denoted by the $L_{d}$ with the largest number was represented as $S_{D_{\text {m }} \text { max }}$. A semicircle was marked with the length $L_{D_{j} \max }$ of the line segment of $S_{D_{j} \max }$ as the radius.

$$
L_{D_{j} \max }=N_{d} L_{d}, \quad N_{d} \text { was the constant. }
$$

For each group of joints $D_{j}$, the average direction $\overline{D_{j}}$ was used as the azimuth angle, and a mark was made on the semicircle. From the center of the circle to the radius of the circle marking point, a point $L_{D_{j}}$ was determined according to the number of $S_{D_{j}}$ in the group and the scale $L_{d}$.

$$
L_{D_{j}}=\frac{S_{D_{j}}}{S_{D_{j} \max }} \cdot L_{D_{j} \max }
$$

e $L_{D_{j}}$ and $L_{D_{j+1}}$ were connected sequentially. If a certain group of joint numbers was zero, the connection would return to the center of the circle and then be connected to the next group from the center of the circle.

A tendency rose diagram of joints was drawn.

\section{Engineering Case Application}

The engineering case used in this study was a rock mass area on a $1300 \mathrm{~m}$ platform on the south slope of the Anjialing Coal Mine, as shown in Figure 2. Anjialing Coal Mine was located in the south-central of Pingshuo mining area in Shuozhou City, Shanxi Province, China. The topography of the mining area was a typical loess plateau. Occurrence strata in the mining area were mainly Quaternary loess, Tertiary laterite, and Carboniferous bedrock. The contact relationship between various levels was unconformable contact. The $1300 \mathrm{~m}$ platform was located in the middle section of the slope, and the platform height was 12 meters. The overall slope angle was $75^{\circ} \sim 85^{\circ}$, and the mining method was steep slope mining.

The geological survey revealed that the rock mass had an obvious stratum. The lithology of the rock formations was mainly mudstone and sandstone with severe weathering. The rock mass was dry and there was basically no water seepage phenomenon. A large number of joints were developed in the rock mass with good penetration. The cutting effect of the structural surface on the surface of the rock mass was very obvious.

3.1. Application of the Data Import Module. A field structural surface measurement was carried out on a rock mass of $1300 \mathrm{~m}$ platform on the southern slope of Anjialing Coal Mine by using the method of on-site measurement. Then the measured structural surface data was automatically imported into the software system.

\subsection{Application of the Calculation Module of Fuzzy Equivalent} Clustering Algorithm. According to the data information of the imported structural plane, the fuzzy equivalent cluster analysis of the structural plane occurrence was realized based on the fuzzy equivalent clustering algorithm. Through cluster analysis, the structural planes were divided into 3 groups. Among them, joint group 1 obtained a total of 30 structural planes, joint group 2 obtained a total of 38 structural planes, and joint group 3 obtained a total of 57 structural planes. Limited by the length of the article, only 20 structural information of each group of joints are listed in Table 1.

3.3. Application of the Pole Diagram Drawing Module. According to the clustering results and the grouping results of the structural planes, the lower hemisphere equal-angle projection method was used to draw the pole diagram of the structural planes, as shown in Figure 3. 


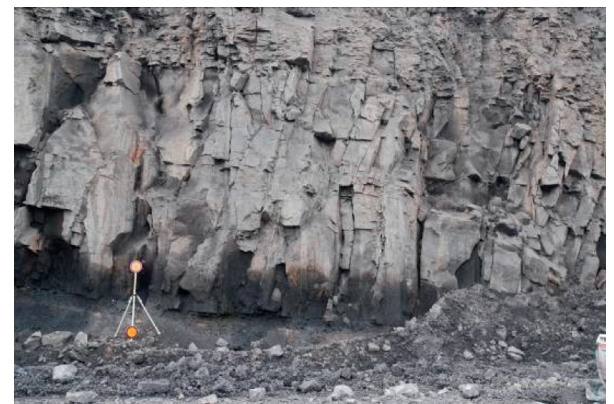

Figure 2: A rock mass on the $1300 \mathrm{~m}$ platform on the south slope of Anjialing Coal Mine.

TABLE 1: The grouping results of structural planes.

\begin{tabular}{|c|c|c|c|c|c|c|c|c|c|}
\hline \multirow{2}{*}{ Joint number } & \multicolumn{3}{|c|}{ Joint group 1} & \multicolumn{3}{|c|}{ Joint group 2} & \multicolumn{3}{|c|}{ Joint group 3} \\
\hline & Trace $(\mathrm{m})$ & Tendency $\left({ }^{\circ}\right)$ & $\operatorname{Dip}\left({ }^{\circ}\right)$ & Trace (m) & Tendency $\left({ }^{\circ}\right)$ & Dip $\left(^{\circ}\right)$ & Trace (m) & Tendency $\left({ }^{\circ}\right)$ & Dip $\left({ }^{\circ}\right)$ \\
\hline 1 & 2.19 & 44.25 & 85.70 & 1.52 & 90.21 & 3.868 & 1.29 & 175.25 & 84.81 \\
\hline 2 & 0.60 & 46.54 & 78.29 & 0.72 & 7.47 & 12.42 & 1.47 & 151.53 & 86.65 \\
\hline 3 & 1.30 & 47.13 & 82.12 & 1.02 & 14.66 & 13.20 & 1.77 & 302.71 & 69.77 \\
\hline 4 & 0.71 & 47.30 & 82.74 & 1.28 & 13.85 & 11.22 & 2.20 & 118.51 & 89.38 \\
\hline 5 & 0.96 & 61.98 & 80.69 & 1.28 & 348.49 & 15.17 & 1.06 & 117.33 & 76.51 \\
\hline 6 & 0.55 & 73.86 & 85.44 & 1.44 & 42.77 & 7.562 & 2.77 & 111.34 & 70.22 \\
\hline 7 & 1.18 & 55.50 & 83.58 & 1.84 & 45.78 & 20.88 & 0.93 & 123.62 & 70.34 \\
\hline 8 & 1.13 & 239.261 & 79.18 & 1.60 & 21.67 & 7.340 & 1.68 & 136.58 & 64.04 \\
\hline 9 & 0.84 & 209.75 & 88.41 & 2.10 & 14.23 & 6.34 & 1.61 & 111.95 & 68.09 \\
\hline 10 & 0.61 & 38.72 & 81.07 & 1.43 & 296.96 & 9.09 & 2.16 & 121.79 & 68.19 \\
\hline 11 & 0.65 & 50.66 & 84.85 & 1.22 & 36.78 & 26.18 & 1.91 & 129.41 & 70.32 \\
\hline 12 & 2.60 & 10.06 & 81.34 & 1.83 & 17.38 & 10.16 & 1.69 & 124.32 & 69.31 \\
\hline 13 & 1.13 & 81.01 & 86.39 & 1.31 & 306.85 & 8.95 & 1.21 & 137.30 & 71.91 \\
\hline 14 & 0.62 & 29.95 & 76.48 & 1.55 & 203.61 & 12.55 & 1.48 & 133.73 & 82.22 \\
\hline 15 & 1.10 & 40.02 & 71.00 & 1.79 & 6.5836 & 8.75 & 1.63 & 145.07 & 81.95 \\
\hline 16 & 0.54 & 18.53 & 60.65 & 1.34 & 59.68 & 17.50 & 1.53 & 109.38 & 86.27 \\
\hline 17 & 1.03 & 258.40 & 84.35 & 1.09 & 73.37 & 2.11 & 1.21 & 309.30 & 89.58 \\
\hline 18 & 1.08 & 238.86 & 88.28 & 2.68 & 40.36 & 3.37 & 2.14 & 141.01 & 88.13 \\
\hline 19 & 1.96 & 83.60 & 68.83 & 2.06 & 32.27 & 12.84 & 0.84 & 132.81 & 78.93 \\
\hline 20 & 0.68 & 72.09 & 63.58 & 1.24 & 65.44 & 21.70 & 2.50 & 131.76 & 77.91 \\
\hline
\end{tabular}

3.4. Application of the Statistical Analysis Module and Data Output Module. The statistical analysis was performed on the structural planes after clusterin. The mean and variance of the tendency, dip, trace, spacing, strike, and bridge of each group of structural planes were obtained. The classification information of the occurrence state of the structural plane was output.

Through the statistical analysis of the structural plane, the geometric parameter statistical distribution results of joint group 1 are shown in Figure 4 .

According to the statistical distribution results in Figure 4, the data output module was used to output the classification information of the occurrence of joint group 1 , as shown in Table 2.

Through the statistical analysis of the structural plane, the geometric parameter statistical distribution results of joint group 2 are shown in Figure 5.

According to the statistical distribution results in Figure 5, the data output module was used to output the classification information of the occurrence of joint group 2, as shown in Table 3.
Through the statistical analysis of the structural plane, the geometric parameter statistical distribution results of joint group 3 are shown in Figure 6.

According to the statistical distribution results in Figure 6, the data output module was used to output the classification information of the occurrence of joint group 3, as shown in Table 4.

3.5. Application of the Drawing Module of Tendency, Dip, and Strike Rose Diagrams. According to the rose diagram drawing method, the tendency, dip, and strike rose diagram of each group of joints were drawn, as shown in Figures 7-9.

\section{Discussion}

The paper combined fuzzy equivalent clustering algorithm, rose diagram drawing method, and $\mathrm{C}++$ language and developed a drawing system for the pole diagram and rose diagram of rock mass structural planes. The system contained 8 modules, which could be easily applied to engineering. 


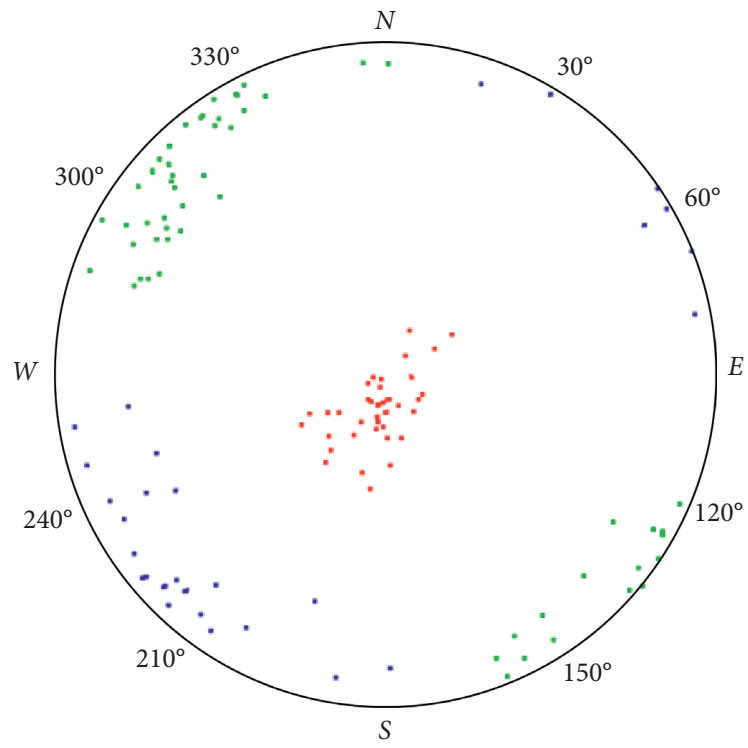

Joint group 1

Joint group 2

Joint group 3

Figure 3: The pole diagram of the structural plane.

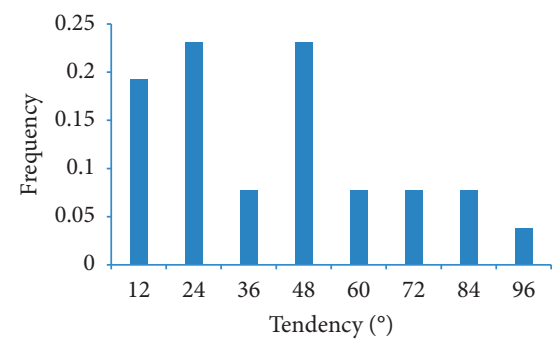

(a)

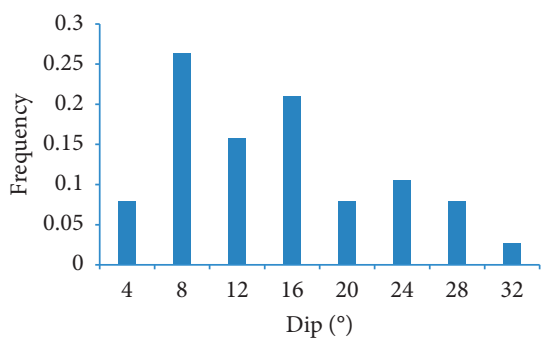

(b)

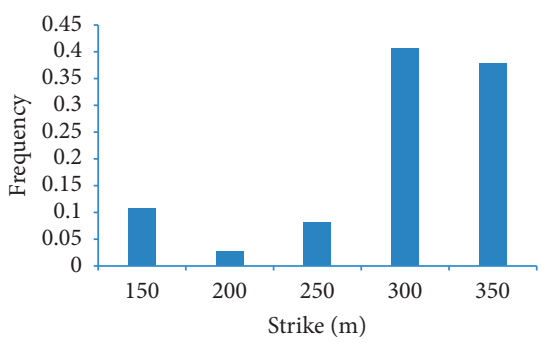

(c)

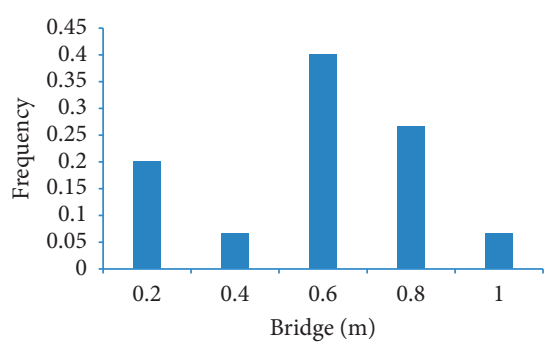

(d)

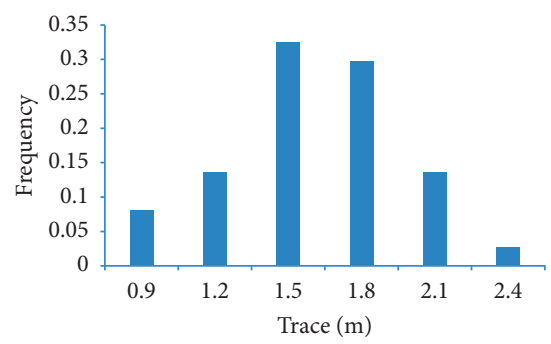

(e)

Figure 4: The results of the statistical distribution of geometric parameters of joints group 1. The distribution rate of (a) tendency, (b) dip, (c) strike, (d) bridge, and (e) trace.

TABLE 2: The geometrical parameters and distribution of the discontinuities.

\begin{tabular}{lcccccc}
\hline Joint group & Eigenvalues & Tendency $\left({ }^{\circ}\right)$ & Dip $\left({ }^{\circ}\right)$ & Strike $\left(^{\circ}\right)$ & Bridge $(\mathrm{m})$ & Trace $(\mathrm{m})$ \\
\hline \multirow{3}{*}{ Joint group 1 } & Mean & 35.09 & 12.79 & 270.15 & 0.48 & 1.42 \\
& Standard deviation & 24.83 & 7.09 & 60.36 & 0.21 & 0.34 \\
& Distribution rate & $\begin{array}{c}\text { Lognormal } \\
\text { distribution }\end{array}$ & $\begin{array}{c}\text { Lognormal } \\
\text { distribution }\end{array}$ & $\begin{array}{c}\text { Lognormal distribution } \\
\end{array}$ & Normal distribution & Normal distribution \\
\hline
\end{tabular}




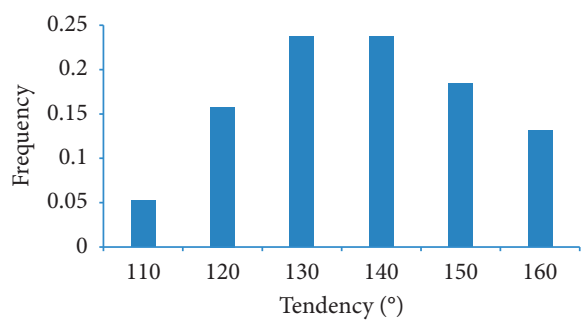

(a)

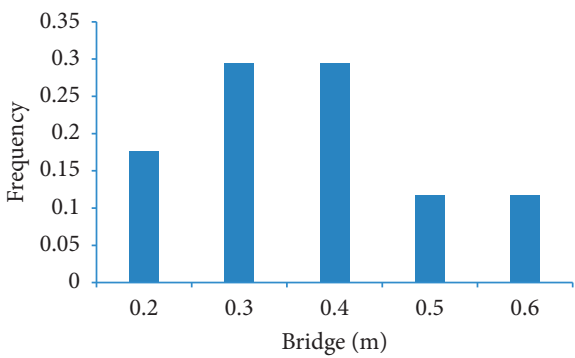

(d)

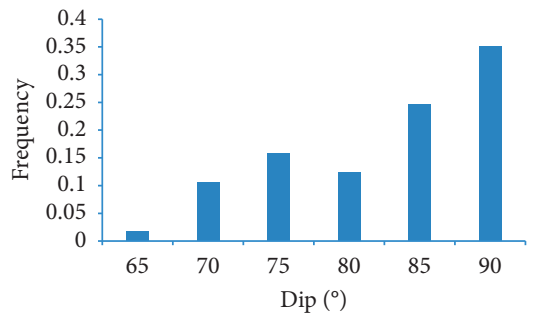

(b)

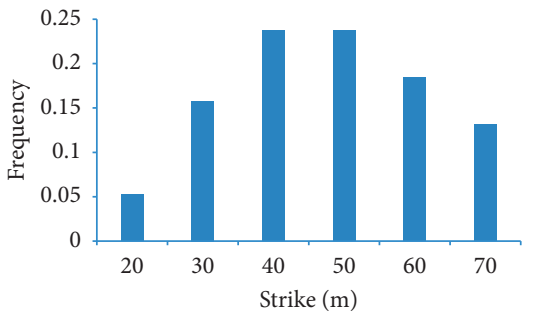

(c)

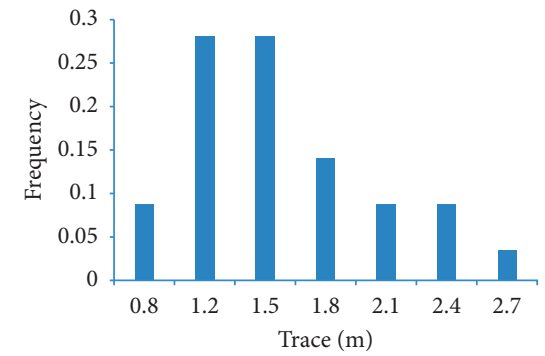

(e)

Figure 5: The results of the statistical distribution of geometric parameters of joints group 2. The distribution rate of (a) tendency, (b) dip, (c) strike, (d) bridge, and (e) trace.

Table 3: The geometrical parameters and distribution of the discontinuities.

\begin{tabular}{|c|c|c|c|c|c|c|}
\hline Joint group & Eigenvalues & Tendency $\left({ }^{\circ}\right)$ & $\operatorname{Dip}\left({ }^{\circ}\right)$ & Strike $\left({ }^{\circ}\right)$ & Bridge $(\mathrm{m})$ & Trace $(\mathrm{m})$ \\
\hline \multirow{3}{*}{ Joint group 2} & Mean & 131.96 & 80.02 & 41.96 & 0.32 & 1.41 \\
\hline & Standard deviation & 13.53 & 7.44 & 13.53 & 0.13 & 0.5 \\
\hline & Distribution rate & $\begin{array}{c}\text { Normal } \\
\text { distribution }\end{array}$ & $\begin{array}{c}\text { Negative exponential } \\
\text { distribution }\end{array}$ & $\begin{array}{c}\text { Normal } \\
\text { distribution }\end{array}$ & Lognormal distribution & Lognormal distribution \\
\hline
\end{tabular}

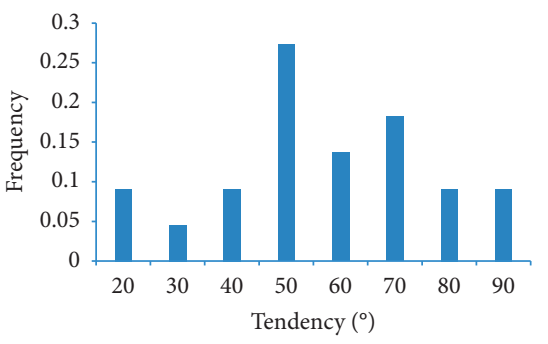

(a)

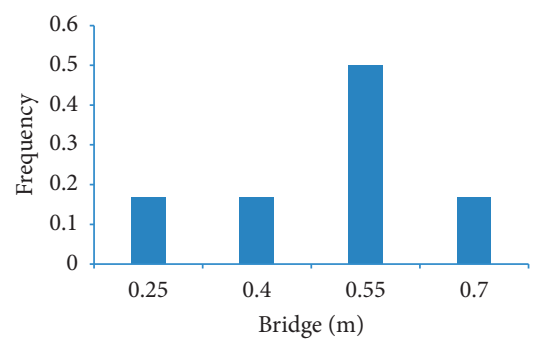

(d)

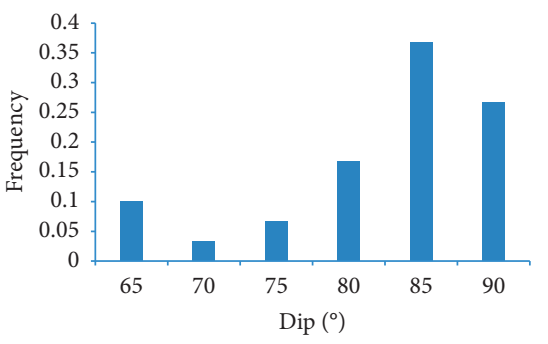

(b)

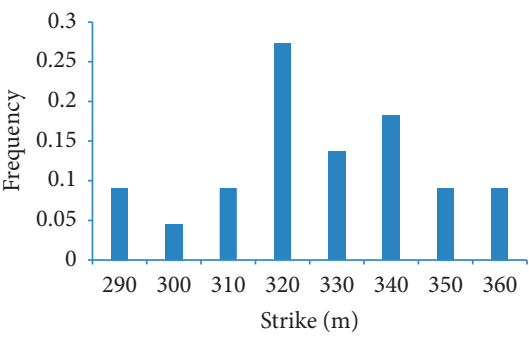

(c)

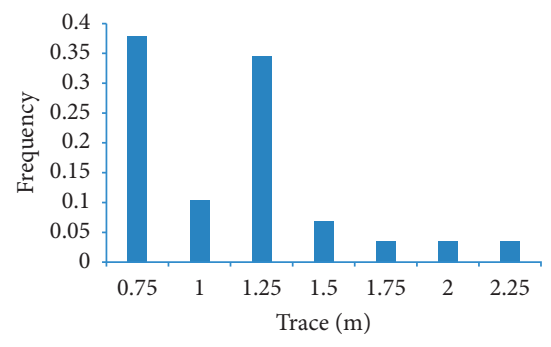

(e)

Figure 6: The results of the statistical distribution of geometric parameters of joints group 3. The distribution rate of (a) tendency. (b) dip, (c) strike, (d) bridge, and (e) trace. 
TABle 4: The geometrical parameters and distribution of the discontinuities.

\begin{tabular}{ccccccc}
\hline Joint group & Eigenvalues & Tendency $\left(^{\circ}\right)$ & Dip $\left(^{\circ}\right)$ & Strike $\left({ }^{\circ}\right)$ & Bridge $(\mathrm{m})$ & Trace $(\mathrm{m})$ \\
\hline \multirow{4}{*}{ Joint group 3 } & Mean & 51.11 & 79.83 & 321.11 & 0.42 & 1.0 \\
& Standard deviation & 18.84 & 7.9 & 18.84 & 0.15 & 0.41 \\
& Distribution rate & Normal & Lognormal & Normal & Normal distribution & Negative exponential distribution \\
& & distribution & distribution & distribution & Normal & \\
& & &
\end{tabular}

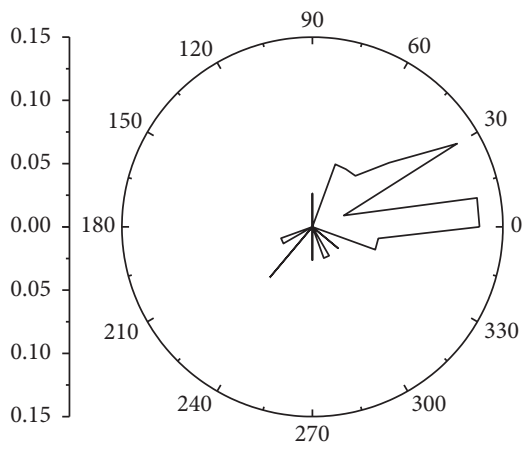

(a)

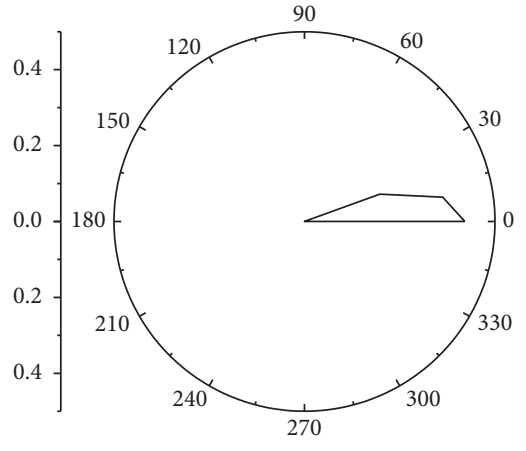

(b)

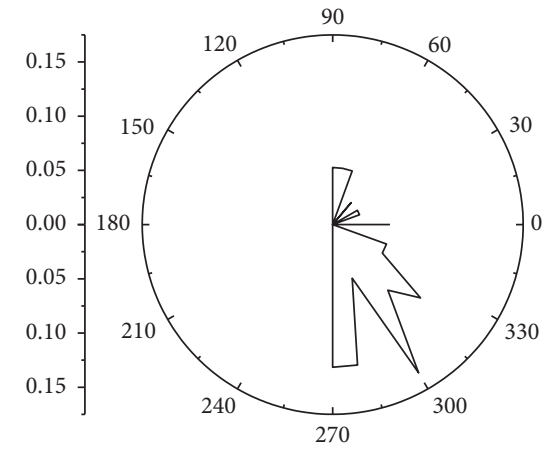

(c)

Figure 7: Rose diagram of joint group 1. (a) The tendency rose diagram. (b) The dip rose diagram. (c) The strike rose diagram.

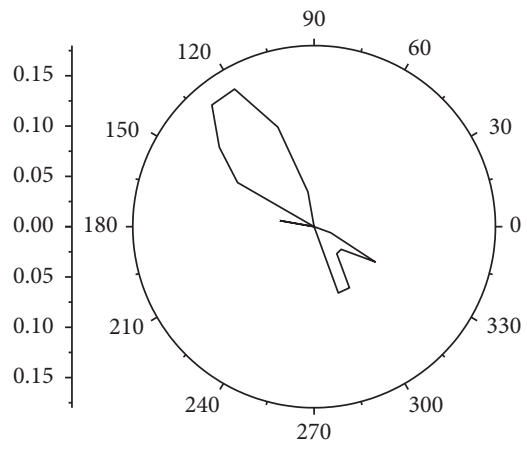

(a)

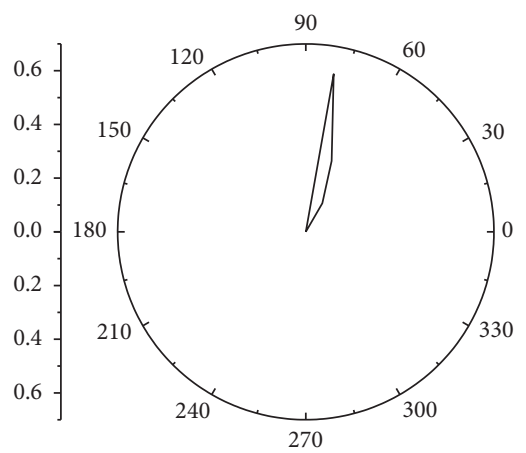

(b)

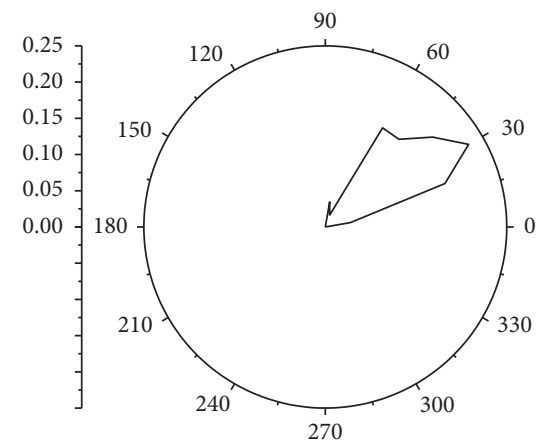

(c)

FIgURE 8: Rose diagram of joint group 2. (a) The tendency rose diagram. (b) The dip rose diagram. (c) The strike rose diagram.

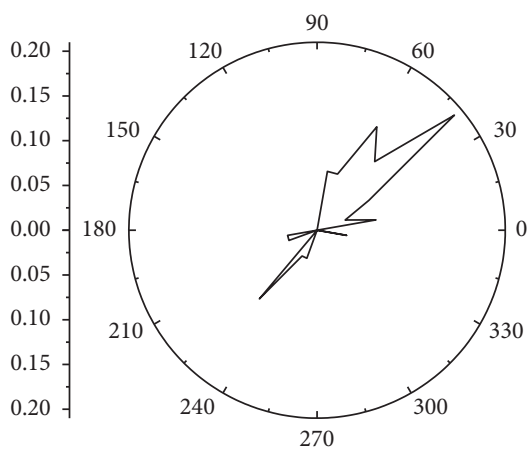

(a)

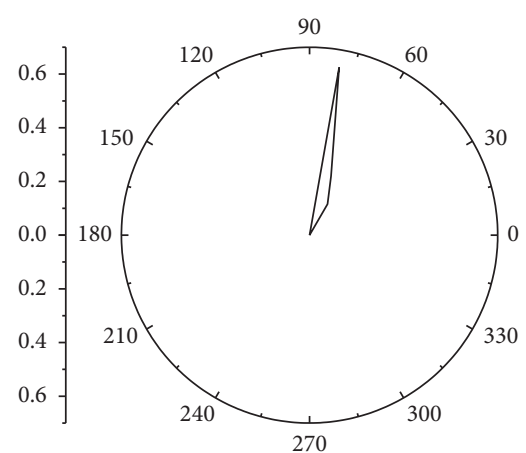

(b)

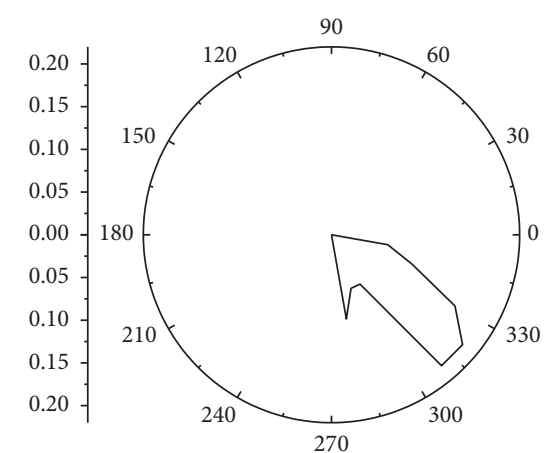

(c)

Figure 9: Rose diagram of joint group 3. (a) The tendency rose diagram. (b) The dip rose diagram. (c) The strike rose diagram. 
The data import module can realize the recognition and import of the structural plane, which solves the difficulty of handling the complex structural planes on-site and reduces the inefficient and repetitive manual operation at the same time.

The calculation module of the fuzzy equivalent clustering algorithm realizes the rapid grouping of structural planes. This module is fast and efficient in processing the structure plane grouping, which saves a lot of processing time.

The pole diagram drawing module can accurately obtain the occurrence distribution and data of the structural plane. This module overcomes the unobjectivity of grouping brought by personal experience and reflects the actual distribution characteristics of the structure data.

The statistical analysis module can perform statistical analysis on the mean value, variance, and probability distribution form of the geometric parameters of the structural plane. This module solves the problems of large workload and high repeatability in the statistical process, which improves work efficiency.

The data output module outputs the grouping and statistical analysis results of the structure plane.

The strike rose diagram drawing module, trending rose diagram drawing module, and dip rose diagram drawing module can realize large-scale repetitive drawing of the trend of multiple sets of structural planes, which reduces the workload and improves the work efficiency.

At present, there are many articles and methods in the treatment of rock mass structural planes, but these articles and methods are often research and analysis on a certain part of the structural plane processing process, and there is no systematic and intelligent structural plane processing system. In the application process, due to the complexity and esoteric content, there are many program algorithms involved, which will cause users to be unable to effectively implement a series of process operations from data import to analysis processing and graphic drawing of structure plane data. It will be more difficult to apply, especially for beginners or laymen. The processing of structural plane data is the most basic and most important link in the analysis of rock mass mechanics. If ordinary geological practitioners are not able to do simple analysis and application of structural plane data, it will greatly reduce their enthusiasm for practicing.

Therefore, this paper has developed a drawing system for the pole diagram and rose diagram of rock mass structural planes, which realizes the digital process operation of the structural plane data import, classification, drawing, statistical analysis, and output. The research and development of this system are beneficial to the processing of the structural surface in the field.

\section{Conclusion}

The paper combined fuzzy equivalent clustering algorithm, rose diagram drawing method, and $\mathrm{C}++$ language and developed a drawing system for the pole diagram and rose diagram of rock mass structural planes, which realized the rapid analysis and drawing of structural planes. The system included the import of data, the fuzzy equivalent clustering grouping, and the drawing of pole diagram, which realized the rapid cluster analysis of the geometric parameters of structural planes. The system included statistical analysis and a data output module, which realized the rapid acquisition and analysis of the probability distribution form of the geometric parameters of the structural plane. The system included a striking rose diagram, a trending rose diagram, and a dip rose diagram module, which realized the rapid drawing and analysis of the rose diagram of the structural plane. Finally, the practicality and convenience of the system were verified through the application of engineering case analysis.

\section{Data Availability}

The data used to support the findings of this study are available from the corresponding author upon request.

\section{Conflicts of Interest}

The author declares that there are no conflicts of interest regarding the publication of this paper.

\section{Acknowledgments}

The author appreciates the support of Zhejiang Collaborative Innovation Center for Prevention and Control of Mountain Geological Hazards (PCMGH-2017-Y-05) and Key Laboratory of Rock Mechanics and Geohazards of Zhejiang Province (ZGRMG-2019-07).

\section{References}

[1] K. Zhao, Q. Wang, Y. Yan et al., "Geological structural surface evaluation model based on unascertained measure," Geofluids, vol. 2019, Article ID 3547384, 7 pages, 2019.

[2] H. I. Chaminé, M. J. Afonso, L. Ramos et al., Scanline Sampling Techniques for Rock Engineering Surveys: Insights from Intrinsic Geologic Variability and Uncertainty, Springer International Publishing, Berlin, Germany, 2015.

[3] W. Xu, Y. Zhang, X. Li et al., "Extraction and statistics of discontinuity orientation and trace length from typical fractured rock mass: a case study of the Xinchang underground research laboratory site, China," Engineering Geology, vol. 269, Article ID 105553, 2020.

[4] S. Manzoor, S. Liaghat, A. Gustafson, D. Johansson, and H. Schunnesson, "Establishing relationships between structural data from close-range terrestrial digital photogrammetry and measurement while drilling data," Engineering Geology, vol. 267, Article ID 105480, 2020.

[5] P. Wang, T. Qin, Z. Huang et al., "Fast identification of geometric properties of rock discontinuities based on 3D point cloud," Chinese Journal of Rock Mechanics and Engineering, vol. 40, no. 3, pp. 503-519, 2021, in Chinese.

[6] G. M. Dong, L. C. Shu, M. M. Wang et al., "Application of fuzzy information diffusion method in fissure structural plane analysis," Journal of Central South University (Science and Technology), vol. 42, no. 8, pp. 2493-2498, 2011.

[7] Z. Tao, Y. Liu, C. Zhu et al., "Comprehensive engineering geological analysis on large-scale anti-dip slopes: a case study 
of changshanhao opencast gold mine in China," Geotechnical and Geological Engineering, vol. 39, no. 5, pp. 1-20, 2020.

[8] X. Wei, Y. Guo, H. Cheng et al., "Rock mass characteristics in beishan, a preselected area for China's high-level radioactive waste disposal," Acta Geologica Sinica, vol. 93, no. 2, pp. 116-126, 2019.

[9] Y. U. Jifeng, L. I. Zhenghong, and X. Chen, "Automatic plotting of rose diagram with geological data and its application," Journal of Shandong University of Science and Technology (Natural Science), vol. 36, no. 3, pp. 1-8, 2017.

[10] S. Li, H. Liu, L. Li et al., "A quantitative method for rock structure at working faces of tunnels based on digital images and its application," Chinese Journal of Rock Mechanics and Engineering, vol. 36, no. 1, pp. 1-9, 2017, in Chinese.

[11] K. Zhao, Q. Wang, J. Wang et al., "Structural plane characteristics and stability of broken surrounding rocks," China Safety Science Journal, vol. 29, no. 9, pp. 138-143, 2019.

[12] W. Zhong, Z. Y. Tan, and L. Qiao, "Stability analysis of rock slope based on preferred structural plane," Advanced Materials Research, vol. 243-249, pp. 2254-2258, 2011.

[13] H. Li, X. Liu, T. Li, and R. Gan, "A novel density-based clustering algorithm using nearest neighbor graph," Pattern Recognition, vol. 102, Article ID 107206, 2020.

[14] Q. Hou, Y. Rui, S. Du et al., "A method for clustering orientation data of discontinuities of rock mass based on netting algorithm," Chinese Journal of Rock Mechanics and Engineering, vol. 39, no. 1, pp. 2871-2881, 2020, in Chinese.

[15] X. Li, Z. Wang, P. Kang et al., "Ant colony ATTA clustering algorithm of rock mass structural plane in groups," Journal of Central South University, vol. 21, no. 2, 2014.

[16] L. I. Jun, H. U. Bin, K. Zhu et al., "An improved fast clustering analysis method for attitude of structural plane in rock mass," Journal of Yangtze River Scientific Research Institute, vol. 33, no. 8, pp. 96-99, 2016.

[17] J. Guo, S. Liu, P. Zhang et al., "Towards semi-automatic rock mass discontinuity orientation and set analysis from $3 \mathrm{D}$ point clouds," Computers and Geosciences, vol. 103, 2017.

[18] H. Bei, Y. Mao, W. Wang, and X. Zhang, "Fuzzy clustering method based on improved weighted distance," Mathematical Problems in Engineering, vol. 2021, Article ID 6687202, 11 pages, 2021. 